\title{
Deterministic Quantum Mechanics: Classical nuclear motion explains chemical reactions and spectra
}

\author{
Erik Rohloff ${ }^{1}$, Dominik Rudolph ${ }^{1}$, Onno Strolka ${ }^{1}$, and Irmgard Frank ${ }^{1}$ \\ ${ }^{1}$ Leibniz Universität Hannover
}

October 22, 2021

\begin{abstract}
Is a classical description of nuclear motion sufficient when describing chemical reactions? The present paper investigates some phenomena that were previously attributed to nuclear quantum effects. The aim is to show that these phenomena can be modelled with traditional Car-Parrinello molecular dynamics, that is, with a method which treats nuclear motion classically. We find that no additional paradigm is needed for describing chemical reactions. The special reactivity observed for carbenes can be attributed to the special environment represented by a noble gas matrix. Also the infrared spectrum of porphycene is perfectly modelled by traditional Car-Parrinello molecular dynamics. If no more convincing examples are produced, one will stick to deterministic quantum mechanics, as it is the simpler theory which, in addition, is free of paradoxa.
\end{abstract}

\section{Hosted file}

frank.pdf available at https://authorea.com/users/442363/articles/542651-deterministicquantum-mechanics-classical-nuclear-motion-explains-chemical-reactions-and-spectra

\section{Hosted file}

frank.tex available at https://authorea.com/users/442363/articles/542651-deterministicquantum-mechanics-classical-nuclear-motion-explains-chemical-reactions-and-spectra 
figures/Fig1/Fig1-eps-converted-to.pdf 
figures/Fig2/Fig2-eps-converted-to.pdf

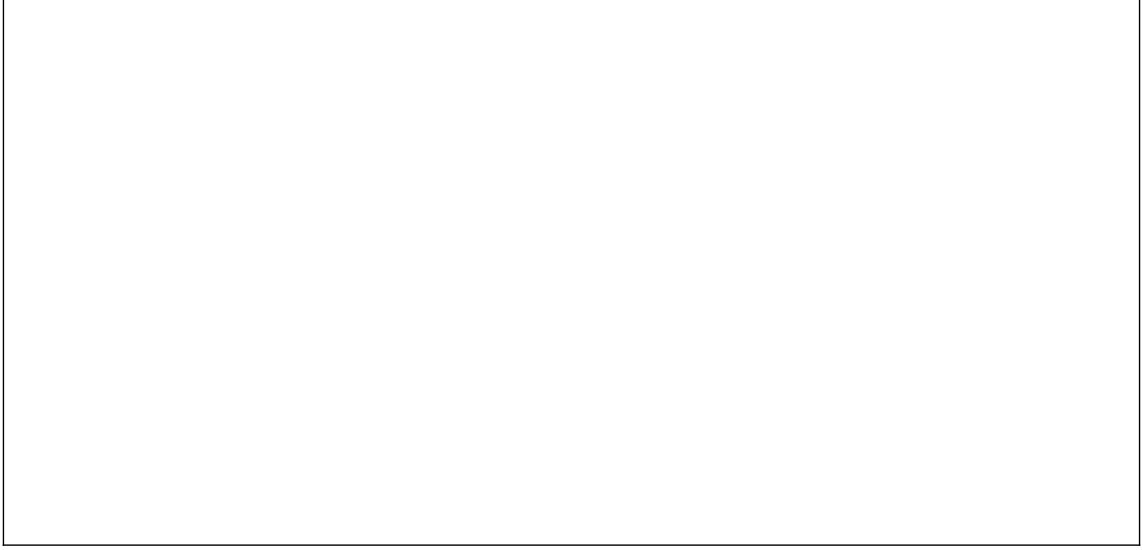

\section{Hosted file}

Fig3.eps available at https://authorea.com/users/442363/articles/542651-deterministicquantum-mechanics-classical-nuclear-motion-explains-chemical-reactions-and-spectra 
figures/Fig4/Fig4-eps-converted-to.pdf 
figures/Fig5/Fig5-eps-converted-to.pdf 
figures/Fig6/Fig6-eps-converted-to.pdf 
figures/Fig7/Fig7-eps-converted-to.pdf 
figures/Fig8/Fig8-eps-converted-to.pdf 